\title{
XLVI. On atmospheric refraction
}

\section{Rev. R. Murphy M.A.}

To cite this article: Rev. R. Murphy M.A. (1842) XLVI. On atmospheric refraction, Philosophical Magazine Series 3, 20:131, 310-312, DOI: 10.1080/14786444208650574

To link to this article: http://dx.doi.org/10.1080/14786444208650574

册 Published online: 01 Jun 2009.

Submit your article to this journal 준

LII Article views: 2

Q View related articles $₫$ 
my doubts public, and shall be thankful if by doing so I should be so fortunate as to elicit an explanation from some of your more experienced correspondents.

I am, Sir, your obedient Servant,

January 1, 1842.

S. Earnshaw.

XLVI. On Atmospheric Refraction. By the Rev. R. MuRPHY, M.A., \&c.*

\section{§ 1. Hypothesis.}

THE refracting power of the atmosphere is a function of its distance from the centre of the earth, and tends in the direction of that centre. The curve described by a luminous ray in its passage through the air is governed by the usual laws of the trajectories of bodies acted on by centripetal forces.

$\$ 2$.- Let the velocity of light entering the atmosphere $=1$, and $v$ that at which it arrives at the earth's surface.

Let $r$ be the distance of a point in the trajectory of the ray from the earth's centre, and $\phi(r)$ the force acting then on light; we have

$$
v^{2}=1+2 f_{r} \phi(r)
$$

the limits of $r$ are 1 (the earth's radius) and $1+h$ ( $h$ being the height of the atmosphere). Hence $v^{2}$ is constant for all incidences, and we may put $v=1+m$ where $m$ is a certain constant.

$\S 3 .-$ Let $\theta$ be the angle made by the radius vector drawn to the earth's centre when the ray enters the atmosphere with that ray, and $z^{\prime}$ be the apparent zenith distance.

The perpendicular on the ray as it enters the atmosphere drawn from the earth's centre $=(1+h) \sin \theta$, and that as it enters the eye $=\sin z^{\prime}$. These are inversely proportional to the corresponding velocities, viz. 1 and $1+m$; hence

$$
(1+h) \cdot \sin \theta=(1+m) \sin z^{\prime} \text {. }
$$

$\S 4$.- Let the observed body be the moon, at a distance $a$, reckoning in radii of the earth, from the centre.

Let $z$ be its true zenith distance seen from the earth's centre, corresponding to the apparent zenith distance $z^{\prime}$.

Let $p$ be the moon's parallax at that zenith distance, and let $\omega$ be the angle at the moon subtended by the bent trajectory of the luminous ray. Then $p+\omega$ is the angle at the moon made by the radius vector from the earth's centre with the issuing ray, which being the same that enters the atmosphere to reach the eye, we have

$$
a \sin (p+\omega)=(1+h) \cdot \sin \theta \text {. }
$$

* Communicated by the Author. 
The Rev. R. Murphy on Atmospheric Refraction.

Hence by section 3 ,

$$
\sin z^{\prime}=\frac{a}{1+m} \cdot \sin (p+w) .
$$

§5.-Now $z$ being the true zenith distance, $z+p+\omega$ is the angle which the issuing ray makes with the spectator's vertical, and this angle diminished by the refraction $r$ is the apparent zenth distance. Hence

$$
\sin (z+p+\omega-r)=\frac{a}{1+m} \sin (p+w) .
$$

\$ 6.-The right line drawn from the moon to the spectator makes with that drawn to the centre of the earth the angle $p$, and with the spectator's vertical $z+p$; hence

or

$$
\begin{aligned}
& \sin (z+p): \sin p:: a: 1, \\
& \sin (z+p)=a \sin p .
\end{aligned}
$$

Hence

$$
\begin{aligned}
\sin z \cos p & =(a-\cos z) \sin p \\
\tan p & =\frac{\sin z}{a-\cos z} \\
\sec ^{2} p & =\frac{a^{2}-2 a \cos z+1}{(a-\cos z)^{2}} \\
\cos ^{2} p & =\frac{(a-\cos z)}{a^{2}-2 a \cos z+1} \\
\sin ^{2} p & =\frac{\sin ^{2} z}{a^{2}-2 a \cos z+1} .
\end{aligned}
$$

Therefore

$$
\begin{aligned}
\cos p & =\frac{a-\cos z}{\left.\sqrt{\left(a^{2}-2 a \cos z+1\right.}\right)} \\
\sin p & =\frac{\sin z}{\sqrt{ }\left(a^{2}-2 a \cos z+1\right)^{\circ}}
\end{aligned}
$$

\$7.-By section 5 ,

$$
\begin{gathered}
\sin (z+\omega-r) \cdot \cos p+\cos (z+\omega-r) \sin p \\
=\frac{a}{1+m}(\sin \omega \cos p+\cos \omega \sin p) .
\end{gathered}
$$

Hence $\quad \cos p\{(1+m) \sin (z+\omega-r)-a \sin \omega\}$

$$
=\sin p\{a \cos \omega-(1+m) \cos (z+\omega-r)\} \text {. }
$$

Therefore, by section 6 ,

$$
\begin{gathered}
\sin z\{a \cos \omega-(1+m) \cos (z+\omega-r)\} \\
=(a-\cos z)\{(1+m) \sin (z+\omega-r)-a \sin \omega\} .
\end{gathered}
$$

This would be the complete solution of the question, were $\omega$ known.

$\oint 8$. - The angle $\omega$ is a function of the parallax, and is evi- 
dently very small compared with it, being less than the angle at the moon, subtended by a tangent to the ray drawn from the eye and bounded by the atmosphere.

Moreover, $w$ vanishes and changes sign at the sarne time with the parallax $p$, and is therefore of the form $k p+l p^{3}$, $\& c$., where $k, l, \& c$. are constants : for an approximation we may reject the higher powers of $p$ and find $\omega=k p$, where $k$ is a small fraction.

$\$ 9 .-B y$ comparing sections 5 and 6 with this result, the law of atmospheric refraction would be given by the two equations,

$$
\left\{\begin{array}{l}
\sin \{z-r+(1+k) \cdot p\}=a^{\prime} \sin (1+k) p \\
\sin (z+p)=a \sin p
\end{array}\right.
$$

The elimination of $p$ between these equations, and the determination of the constants by observation, would give the approximate formula for the relation between the refraction and true zenith distance, which of course would apply to light coming from any heavenly body, though originally deduced from but one of them.

$\S 10$. - The equations of section 9 may be written in terms of the apparent zenith distance $z^{\prime}$,

$$
\left\{\begin{array}{l}
\sin z^{\prime}=c a \sin (1+k) p \\
\sin \left(z^{\prime}+r-k p\right)=a \sin p,
\end{array}\right.
$$

where $c$ is an absolute constant $=\frac{1}{1+m} ; a$ is arbitrary, but had better be large, and $k$ is a small constant and a function of $a$. The elimination of $p$ gives the relation between $z^{\prime}$ and $r$.

$\S$ 11.-Approximation by rejecting $k^{2} p^{2}, a p^{3}$, \&c., and eliminating

or

$$
\sin z^{\prime}=\frac{\alpha \sin \left(z^{\prime}+r\right)}{1-\frac{2 \beta}{\alpha} \cdot \cos \left(z^{\prime}+r\right)},
$$

$\alpha$ differing but little from unity, and $\beta$ being a small fraction.

R. M.

XLVII. On the Composition of Wolfram. By W. H. Miller, Esq., Professor of Mineralogy in the Unizersity of Cambridge*

THE analyses of wolfram from a number of different lo1 calities by Count Francis Schaffgotsch, show (1.) That the sum of the bases is always found to be larger than in a

* From Poggendorff's Annalen, Band lii. 